\title{
A IMPORTÂNCIA DA CONSCIENTIZAÇÃO DO CONSUMIDOR SOBRE TERMOS ALIMENTARES \\ Projeto de extensão com alunos do ensino fundamental
}

Jennifer Lima Costa
Universidade Federal de Viçosa
Jennifer.costa@ ufv.br

Tayna Jamille Santos

Universidade Federal de Viçosa

tavnaiamille@gmail.com

Patrícia Natalina Santos

Universidade Federal de Viçosa

Raquel Lucena Souza

Universidade Federal de Viçosa

raquel.lucena08@gmail.com

Robledo de Almeida Torres Filho

Universidade Federal de Viçosa robledo.filho@ufv.br

Vanelle Maria da Silva Universidade Federal de Viçosa vanelle.silva@gmail.com

Naiara Barbosa Carvalho

Universidade Federal de Viçosa naieal@vahoo.com.br

\begin{abstract}
Resumo
Esse estudo é baseado no projeto de extensão "Terminologias aplicadas em alimentos: conscientização do consumidor para a sua autonomia no momento da compra", em que o objetivo foi compreender e conscientizar os alunos do ensino fundamental de uma Escola Estadual de Florestal - MG, sobre os termos diet, light, probiótico, prebiótico, irradiado e transgênico. Os temas foram apresentados por meio de palestras e atividades dinâmicas em seis encontros. No primeiro encontro, foi aplicado aos alunos um questionário contendo questões objetivas sobre o significado de cada tema citado acima e outro questionário, elaborado com questões sociodemográficas, de hábitos de consumo e de possíveis doenças na família relacionadas com os mesmos termos, foi entregue para os alunos responderem com o auxílio dos pais em suas casas. O questionário para os alunos foi novamente aplicado após os encontros a fim de avaliar se houve alteração no índice de acertos após as explicações. Posteriormente, foram realizadas três palestras sobre, respectivamente, os temas probiótico e prebiótico, diet e light, e transgênico e irradiado, com a apresentação de cada tema contendo exemplos de alimentos, os benefícios que proporcionam à saúde, definições e o modo de consumo. Ao final de cada palestra era realizado um jogo de perguntas relacionado às definições do tema abordado.
\end{abstract}


Inicialmente, os índices de acerto foram: o probiótico (31\%), seguido por prebiótico e o irradiado (45\%), o light (56\%), o transgênico (58\%), e, por último, o diet (97\%). No questionário final, os índices de acertos foram: light $(71 \%)$, irradiado (63\%), probiótico $(60 \%)$, transgênico (56\%), prebiótico (40\%) e diet (39\%), sendo que o termo light obteve o maior índice de acertos. Isso demonstra que os alunos entenderam os termos, sabiam explicar seus significados e dar exemplos de alimentos. Desse modo, observou-se que os jovens agregaram conhecimento sobre os termos técnicos e símbolos atuais que se encontram presentes nos rótulos de produtos alimentícios e, logo, estão mais conscientes do consumo desses alimentos. Para a equipe do Projeto, o mesmo foi de muita valia, tanto para a bolsista quanto para as voluntárias, pois apesar das dificuldades encontradas no desenvolvimento do trabalho, o mesmo contribuiu para agregar conhecimento e auxiliar na formação acadêmica e pessoal das discentes.

Palavras-chave: Conscientização. Light. Diet. Prebiótico. Probiótico. Irradiado. Transgênico.

\title{
THE IMPORTANCE OF CONSUMER CONSCIOUSNESS ON FOOD TERMS Extension project with elementary school students
}

\begin{abstract}
This study is based in Project of extension "Terminologies applied in food: consumer awareness for their autonomy at the time of purchase", in which the objective was to understand and raise awareness of elementary school students of a State School of Florestal - MG, on the terms diet, light, probiotic, prebiotic, irradiated and transgenic. The themes were presented through lectures and dynamic activities in six meetings. At the first meeting, a questionnaire containing objective questions about the meaning of each topic mentioned above was applied to the students, and another questionnaire, elaborated with sociodemographic questions, consumption habits and possible diseases in the family related to the same terms, was given to the students respond with their parents' help in their homes. The questionnaire for the students was again applied after the meetings in order to evaluate if there was a change in the success rate after the explanations. Subsequently, three lectures on probiotic and prebiotic, diet and light, and transgenic and irradiated subjects were carried out, with the presentation of each topic containing examples of food, the health benefits, definitions and the mode of consumption. At the end of each lecture, a set of questions was asked related to the definitions of the topic addressed. Initially, the probiotic percentages were: probiotic $(31 \%)$, followed by prebiotic and irradiated $(45 \%)$, light $(56 \%)$, transgenic $(58 \%)$, and finally diet $(97 \%)$. In the final questionnaire, the correct answers were: light (71\%), irradiated (63\%), probiotic (60\%), transgenic (56\%), prebiotic (40\%) and diet (39\%) light obtained the highest hit rate. This demonstrates that students understood the terms, knew how to explain their meanings and give examples of foods. Thus, it was observed that the young people added knowledge about the current technical terms and symbols that are present in the labels of food products and, therefore, are more aware of the consumption of these foods. For the Project team, it was very valuable, both for the scholarship recipient and for the volunteers, because despite the difficulties encountered in the development of the work, it contributed to add knowledge and help in the academic and personal formation of the students.
\end{abstract}

Key words: Awareness. Light. Diet. Prebiotic. Probiotic. Irradiated. Transgenic.

\section{LA IMPORTANCIA DE LA CONCIENTIZACIÓN DEL CONSUMIDOR SOBRE TÉRMINOS ALIMENTARIOS Proyecto de extensión con alumnos de enseñanza primaria}

\begin{abstract}
Resumen
Este estudio se basa en el proyecto de ampliación "Terminologías aplicadas a la alimentación: concienciación del consumidor sobre su autonomía al momento de la compra", en el que el objetivo fue comprender y concienciar a los alumnos de primaria de una Escuela Forestal del Estado - MG, sobre los términos dieta, ligero, probiótico, prebiótico, irradiado y transgénico. Los temas se presentaron a través de conferencias y actividades dinámicas en seis reuniones. En la primera reunión se aplicó a los estudiantes un cuestionario que contenía preguntas objetivas sobre el significado de cada tema mencionado anteriormente y se entregó a los estudiantes otro cuestionario, elaborado con aspectos sociodemográficos, hábitos de consumo y posibles enfermedades en la familia relacionadas
\end{abstract}


con los mismos términos. los estudiantes responden con la ayuda de los padres en sus hogares. El cuestionario para estudiantes se aplicó nuevamente después de las reuniones con el fin de evaluar si hubo un cambio en el índice de corrección después de las explicaciones. Posteriormente, se realizaron tres conferencias sobre, respectivamente, los temas probiótico y prebiótico, dieta y light, y transgénico e irradiado, con la presentación de cada tema conteniendo ejemplos de alimentos, los beneficios que brindan a la salud, definiciones y modo de consumo. Al final de cada conferencia se llevó a cabo un juego de preguntas relacionadas con las definiciones del tema abordado. Inicialmente, las tasas de éxito fueron: probiótico (31\%), seguido de prebiótico e irradiado (45\%), ligero (56\%), transgénico (58\%) y, finalmente, dieta (97\%). ). En el cuestionario final, las tasas de éxito fueron: ligero (71\%), irradiado (63\%), probiótico (60\%), transgénico (56\%), prebiótico (40\%) y dieta (39\%), con el término la luz tuvo la tasa de aciertos más alta. Esto demuestra que los estudiantes entendieron los términos, supieron explicar sus significados y dar ejemplos de comida. Así, se observó que los jóvenes suman conocimientos sobre los términos técnicos y símbolos actuales que están presentes en las etiquetas de los productos alimenticios y, por tanto, son más conscientes del consumo de estos alimentos. Para el equipo del Proyecto fue de gran valor, tanto para el becario como para los voluntarios, pues a pesar de las dificultades encontradas en el desarrollo del trabajo, contribuyó a sumar conocimientos y asistir en la formación académica y personal de los estudiantes.

Palabras clave: Conciencia. Light. Diet. Prebiótico. Probiótico. Irradiado. Transgénico. 


\section{INTRODUÇÃO}

A rotulagem é um instrumento de informação e orientação ao consumidor sobre a qualidade e a quantidade dos constituintes nutricionais dos alimentos, permitindo que o indivíduo faça escolhas alimentares apropriadas em sua dieta (JARDIM et al., 2016). A possibilidade de orientação da população quanto ao consumo adequado de alimentos pode corrigir falhas de alimentação, diminuir seus efeitos deletérios e promover o redirecionamento de alimentos pelo setor produtivo (PONTES et al., 2009).

A variedade de produtos nos supermercados tem aumentado nos últimos anos, e, logo, em razão da falta de informação, a maioria da população tem consumido alimentos de forma inadequada. Por exemplo, os termos diet e light, indicados para quem precisa manter dietas restritivas ao açúcar ou está preocupado com a estética e em manter hábitos alimentares saudáveis (VIEIRA e CORNÉLIO, 2018), e os termos irradiados e transgênicos, que estão relacionados a produtos advindos de novas tecnologias. Além desses, os termos probióticos e prebiótico estão associados ao bom funcionamento do organismo. Os probióticos são caracterizados por serem um suplemento alimentar à base de microrganismos vivos que, quando consumidos em quantidades adequadas, afetam beneficamente a saúde do homem. Os prebióticos são utilizados para designar componentes alimentares não digeríveis que afetam beneficamente o hospedeiro, por estimularem seletivamente a proliferação ou atividade de populações de bactérias desejáveis no cólon (MATTA; KUNIGK, 2009).

A educação nutricional é enfatizada pela Política Nacional de Alimentação e Nutrição (BRASIL, 1999) como de suma importância para a geração de hábitos alimentares saudáveis. Ela está vinculada à produção de informações que possam subsidiar os indivíduos a tomar uma melhor decisão no ato da compra. Contudo, há pouca divulgação sobre o significado dos termos veiculados nas embalagens e dos seus modos de consumo (OLIVEIRA; BOCCHINI, 2015). Desse modo, intervenções relacionadas à educação nutricional sobre esses termos são importantes para incentivar o hábito da leitura e interpretação dos rótulos alimentares (CAVADA, 2012).

Os hábitos alimentares são determinados por fatores históricos, sociais, religiosos, econômicos e geográficos. Desse modo, a escola é um ambiente propício para a aplicação de programas de educação em saúde, pois a mesma está inserida em todas as dimensões do aprendizado (ORNELAS, 2006). Ao entender e compreender os rótulos alimentícios na adolescência o indivíduo começa a se interessar pela compra daquilo que consome. Além disso, essa fase é crítica em razão das diversas doenças relacionadas a hábitos alimentares como, obesidade, diabetes e hipertensão. Assim, esse 
momento é oportuno para a educação nutricional a fim de que esse indivíduo possa, não somente comprar e consumir de forma consciente, mas também propagar esse conhecimento para outras pessoas ao seu redor (NUNES; FIGUEIROA; ALVEZ, 2007; SANTOS et al., 2016).

Portanto, o objetivo desse trabalho foi promover o conhecimento e a conscientização dos jovens do ensino fundamental de uma escola pública em Florestal MG sobre termos técnicos e símbolos alimentícios dos rótulos dos produtos, possibilitando o consumo adequado de alimentos denominados funcionais, para fins especiais e produzidos por diferentes tecnologias.

\section{METODOLOGIA}

O projeto de extensão "Terminologias aplicadas em alimentos: conscientização do consumidor para a sua autonomia no momento da compra" foi realizado de março a novembro de 2017, com 88 alunos do oitavo ano do ensino fundamental. O mesmo foi avaliado e aprovado pelo Comitê de Ética da Universidade Federal de Viçosa, processo n. 55445016.4.0000.5153/2017, cumprindo os requisitos para a sua publicação.

No primeiro encontro, o projeto foi apresentado aos alunos, juntamente com o termo de consentimento livre e esclarecido para eles e os responsáveis, já que os participantes eram menores de idade. Em seguida, um questionário estruturado contendo seis questões foi aplicado para avaliar o conhecimento prévio dos alunos sobre os termos diet, light, prebiótico, probiótico, irradiado e transgênico. As questões do questionário (Tabela 1) foram elaboradas com base nos estudos de Santos et al. (2016) e Tezotto-Uliana et al. (2015). Ao final do projeto, o mesmo questionário foi aplicado novamente aos participantes, após a realização de todas as palestras, a fim de comparar o conhecimento inicial e o aprendizado adquirido. Ainda no primeiro encontro, um questionário semiestruturado (Tabela 1), baseado nos estudos citados acima, contendo quatro questões sociodemográficas, três de hábitos de consumo e de possíveis doenças na família que se relacionassem com os termos foi entregue para que fosse respondido com o auxílio dos responsáveis. Os resultados de ambos os questionários foram analisados por meio da estatística descritiva (análise de frequência) no programa SPSS 15.0® (Statistical Package for the Social Sciences).

\section{Aulas sobre terminologias}

No segundo, terceiro e quarto encontros foram abordados, respectivamente, os termos probiótico e prebiótico, diet e light, e transgênico e irradiado da seguinte 
maneira: definição do termo, exemplos de alimentos, vantagens e desvantagens de cada tecnologia, curiosidades, notícias recentes, entre outros tópicos importantes sobre essas terminologias. A exposição do conteúdo por data show permitiu apresentar imagens e animações coloridas que atraíram a atenção dos alunos. Os encontros foram realizados na aula de Ciências, sendo 30 minutos de explicação e 20 minutos de dinâmica.

Tabela 1: Resumo das perguntas dos questionários para os alunos e para os pais

\begin{tabular}{|c|c|}
\hline $\begin{array}{l}\text { Perguntas realizadas no questionário } \\
\text { para os alunos }\end{array}$ & $\begin{array}{l}\text { Perguntas realizadas no questionário para os } \\
\text { pais }\end{array}$ \\
\hline Qual a definição correta para o termo diet? & Gênero do aluno. \\
\hline $\begin{array}{l}\text { Qual a definição correta para o termo } \\
\text { light? }\end{array}$ & Idade do aluno. \\
\hline $\begin{array}{l}\text { Qual a definiç̧ão correta para o termo } \\
\text { probiótico? }\end{array}$ & Renda familiar mensal. \\
\hline $\begin{array}{l}\text { Qual a definiç̧ão correta para o termo } \\
\text { prebiótico? }\end{array}$ & Quantidade de pessoas dependentes da renda. \\
\hline $\begin{array}{l}\text { Qual a definição correta para o termo } \\
\text { transgênico? }\end{array}$ & $\begin{array}{l}\text { Consome algum alimento que seja diet, light, } \\
\text { probiótico, prebiótico, transgênico ou irradiado? }\end{array}$ \\
\hline \multirow[t]{2}{*}{$\begin{array}{l}\text { Qual a definição correta para o termo } \\
\text { irradiado? }\end{array}$} & $\begin{array}{l}\text { Se consome esses produtos, citar no máximo três } \\
\text { exemplos que possui em casa. }\end{array}$ \\
\hline & $\begin{array}{l}\text { Algum membro da família possui alguma doença } \\
\text { como diabetes, obesidade, pressão alta, entre } \\
\text { outras? }\end{array}$ \\
\hline
\end{tabular}

Fonte: Dados da Pesquisa (2017).

\section{Dinâmica: Jogo de perguntas}

Ao final de cada palestra, um jogo de perguntas foi realizado para fixar o conteúdo abordado e sanar dúvidas. Cerca de cinco perguntas abertas eram realizadas sobre o tema. Um aluno se voluntariava a responder a pergunta e poderia escolher um colega para ajudá-lo. A dupla tinha um tempo cronometrado para responder, e, caso cumprisse a tarefa, era somado um ponto. Se não conseguisse acertar a resposta, poderia pedir ajuda para o restante da turma valendo meio ponto. Desse modo, o desempenho dos alunos foi avaliado pelos acertos (\%) das perguntas de cada encontro.

\section{Cartilha}

No último encontro, uma cartilha sobre o conteúdo do projeto foi apresentada. Dois exemplares foram apresentados aos alunos e disponibilizados na biblioteca. A cartilha foi elaborada com ferramenta web Pixton no formato de quadrinhos para atrair a atenção dos jovens e facilitar a compreensão do conteúdo. A história ocorreu em um supermercado e se baseou na conversa entre um profissional da área e duas crianças 
curiosas, que buscavam entender e conhecer mais sobre as terminologias abordadas.

\section{RESULTADOS E DISCUSSÃO: Questionários aplicados aos alunos}

O questionário referente ao significado dos termos, que foi aplicado no primeiro e último encontro, foi respondido por 88 alunos, sendo 43 do sexo masculino e 45 do sexo feminino, com idade média de 13,6 $\pm 1,5$.

No questionário inicial, os índices de acerto antes da realização das palestras foram: diet (97\%), transgênico (58\%), light (56\%), irradiado (45\%), prebiótico (45\%) e probiótico (31\%) (Figura 1). Diet, light e transgênico apresentaram mais acertos que erros. Apesar de light ser mais comum no cotidiano, esse obteve menos acertos que o termo transgênico. Isso pode ter ocorrido por escolha aleatória da resposta ou por transgênicos estarem sendo muito abordado ultimamente em diversos meios de comunicação, como televisão e internet (SANTOS et al., 2016; TEZOTTO-ULIANA et al., 2015).

Figura 1: Comparação de acertos (\%) por termo antes e após os encontros explicativos.

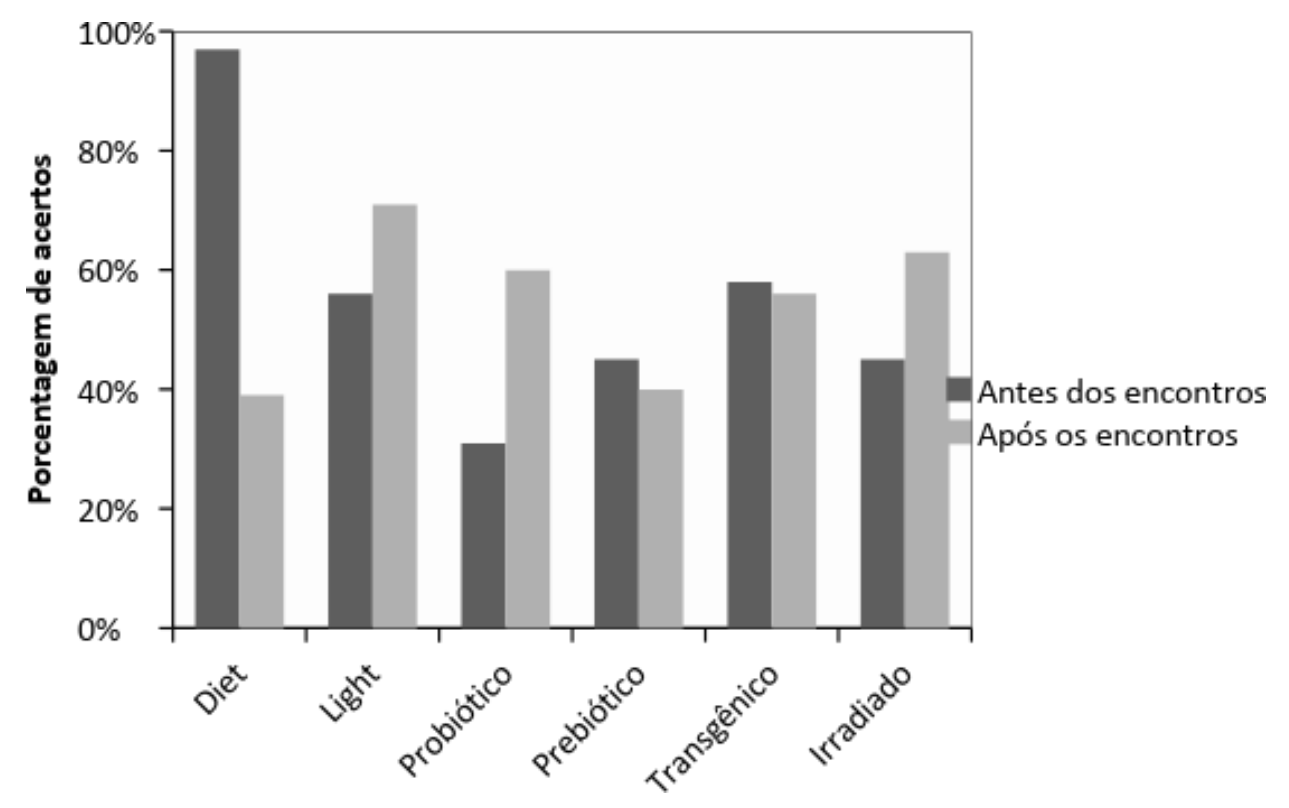

Fonte: Dados da Pesquisa (2017).

No questionário final, os índices de acerto após a realização das palestras foram: light $(71 \%)$, seguido por irradiado (63\%), probiótico (60\%), transgênico (56\%), prebiótico (40\%) e diet (39\%). Light, irradiado e probiótico apresentaram aumento de acertos, com destaque para o probiótico apresentou aumento de quase $30 \%$ de acertos. Transgênico e prebiótico tiveram uma redução de 2 a 5\% nos acertos, o que demonstra 
pouca diferença entre o conhecimento dos participantes antes e após as palestras. Por outro lado, diet apresentou uma redução de $58 \%$.

No geral, houve um aumento do conhecimento dos alunos, principalmente em relação à definição de probiótico. Em relação aos termos que sofreram diminuição de acertos, isso pode ter ocorrido em razão de "chutes" no questionário inicial, principalmente em relação ao termo diet, pois é um termo que apresentou um elevado índice de acertos inicialmente, porém, foi observado que quase ninguém sabia ao certo o seu significado durante a palestra. Um fator não avaliado que pode ter afetado negativamente o resultado final foi que alguns alunos faltaram ou saíram durante a palestra para fazer provas de recuperação, por exemplo.

Santos (2016) realizou um estudo em Fortaleza (CE), a fim de verificar o conhecimento de 18 consumidores, com idade média de 27,5 anos, quanto aos termos light e diet. Esse constatou que $22 \%$ dos participantes conheciam os termos. O autor considerou esse resultado como negativo, pois, apesar de serem termos presentes nos rótulos de vários alimentos, a maioria dos consumidores os desconhecem. No presente trabalho, os alunos obtiveram um melhor desempenho. No entanto, esses resultados ainda não podem ser considerados satisfatórios, indicando, portanto, a necessidade de maior esclarecimento e divulgação sobre essas terminologias.

\section{Questionário aplicado aos alunos juntamente com os pais}

Apenas 13 questionários foram respondidos. Esse pequeno retorno ocorreu por causa do mesmo ter sido enviado para casa, pois alguns alunos o perderam, outros não conseguiram responder com os pais ou o esqueciam em casa. Isso foi uma das dificuldades de executar essa parte do projeto. Nos questionários respondidos, a maioria dos alunos era do sexo feminino (92\%) e apenas um do sexo masculino (8\%), com idade entre doze e treze anos. A faixa de renda mensal familiar da maioria desses alunos (62\%) se situa entre um a cinco salários mínimos ( $\mathrm{R} \$ 937,00$ a $\mathrm{R} \$ 4.695,00)$, sendo que, em média, quatro pessoas dependem dessa renda.

Os alimentos relacionados aos termos apresentaram o seguinte consumo: probiótico (62\%), seguido por prebiótico (54\%), transgênico (46\%), light (39\%), irradiado $(23 \%)$ e, por último, diet $(8 \%)$.

Isso demonstra a tendência da indústria de alimentos de produzir iogurtes e leites fermentados funcionais. Entretanto, apenas iogurtes e leites fermentados contendo culturas probióticas são funcionais e os consumidores, em geral, não sabem distinguir 
quais são os produtos lácteos funcionais. Além disso, há dificuldade em seguir as recomendações de quantidade e frequência de consumo para assegurar os benefícios funcionais e, logo, beneficiar a saúde do consumidor (ANTUNES et al., 2007).

Em relação aos exemplos de alimentos, o termo light, os alimentos mais citados foram iogurte e manteiga (29\%), suco e maionese (14\%). Para o termo diet, refrigerante, manteiga e sorvete foram igualmente $(33 \%)$ mencionados. Para o termo probiótico, o iogurte foi o alimento mais citado (38\%), seguido de queijo (25\%), leite fermentado (19\%), e abacate, limão e coalhada com (6\%). Portanto, para esse último termo, foi possível observar que, apesar deste ter sido apontado como o mais consumido, alguns respondentes indicaram exemplos que não são alimentos probióticos.

Já para o termo prebiótico, alguns dos alimentos citados foram alho e cebola (18\%); banana e aveia (12\%), e mel e aspargo (6\%). Em relação a esse termo, todos os alimentos citados estavam corretos, pois os mesmos possuem compostos bioativos, tais como oligofrutose (FOS) e, ou inulina (I) (FORTES e MUNIZ, 2009). Para o termo transgênico, os exemplos citados foram: amido de milho (19\%); pipoca, óleo e fubá (13\%); soja, salsicha, milho e farinha de milho (6\%). Neste caso, foi percebido que a maioria dos alimentos citados foi o milho e derivados (56\%). Esse resultado pode estar relacionado ao fato de quase todo o milho consumido no país (>90\%) ser transgênico. Além disso, essa cultura é uma das principais cultivadas no país com o grão geneticamente modificado, perdendo apenas para a soja, que também apareceu entre os termos citados (CURY, 2016).

Por fim, para o termo irradiado, os termos foram apenas três, sendo conservas $(50 \%)$ e carne de porco e arroz (25\%). Ornellas et al. (2006) evidenciaram que a falta de informação sobre a tecnologia de irradiação é um fator limitante do seu uso mais frequente na indústria alimentícia brasileira, pois $89 \%$ dos entrevistados consumiriam alimentos irradiados se soubessem que a irradiação aumenta a segurança alimentar.

Em relação a ocorrência de doenças, a maioria afirmou que eles e outros familiares não possuíam nenhuma das doenças. A pressão alta apresentou maior incidência (54\%), obesidade (23\%), e outras doenças (15\%) apresentaram menores ocorrências. A pequena indecência de diabetes coincidiu com o pouco consumo de alimentos diet, no entanto, os alimentos mais consumidos são probióticos e prebióticos, mesmo com nenhum dos entrevistados apresentando síndrome do intestino irritável. Isso demonstra que os benefícios desses alimentos podem ser conhecidos por pessoas que não são portadoras da doença. 


\section{Encontros: palestras sobre os termos}

No segundo encontro, uma palestra foi realizada sobre os termos probiótico e prebiótico. No jogo, somente uma das 13 duplas, "passou” a pergunta "Qual a diferença de probiótico e prebiótico?” para a sala por não saber responder ou ter ficado em dúvida, porém os demais alunos responderam a mesma corretamente. Ainda assim, o conceito foi novamente explicado para não restarem dúvidas. Portanto, pode-se afirmar que a turma teve, em média, $95 \%$ de acerto das perguntas. No terceiro e no quarto encontro, os alunos acertaram todas as perguntas, demonstrando que a aula explicativa se mostrou eficaz (Tabela 2).

Tabela 2: Acertos (\%) das perguntas realizadas após as aulas de cada encontro

\begin{tabular}{|c|c|c|}
\hline Encontro & Perguntas & $\begin{array}{l}\text { Acertos } \\
(\%)\end{array}$ \\
\hline \multirow{5}{*}{$\begin{array}{l}\text { Segundo encontro: } \\
\text { Probiótico e } \\
\text { Prebiótico }\end{array}$} & $\begin{array}{l}\text { 1- Em que alimentos encontram-se os probióticos? Cite pelo } \\
\text { menos dois exemplos. }\end{array}$ & 100 \\
\hline & $\begin{array}{l}\text { 2- Em que alimentos encontram-se os prebióticos? Cite pelo } \\
\text { menos dois exemplos. }\end{array}$ & 100 \\
\hline & 3- Qual a diferença entre probiótico e prebiótico? & 77 \\
\hline & $\begin{array}{l}\text { 4- Como consumir os probióticos para que confiram } \\
\text { benefícios? }\end{array}$ & 100 \\
\hline & 5- Qual o benefício de um simbiótico? & 100 \\
\hline \multirow{5}{*}{$\begin{array}{l}\text { Terceiro encontro: } \\
\text { Diet e Light }\end{array}$} & $\begin{array}{l}\text { 1- Uma nutricionista passou uma nova dieta para um paciente } \\
\text { que quer emagrecer. Quais tipos de alimentos ele deve } \\
\text { consumir? }\end{array}$ & 100 \\
\hline & $\begin{array}{l}\text { 2- Uma pessoa descobriu que está com diabetes e passou a } \\
\text { consumir alimentos light Ela fez certo ou errado? Justifigue }\end{array}$ & 100 \\
\hline & $\begin{array}{l}\text { 3- Uma Engenheira de Alimentos desenvolveu um novo } \\
\text { refrigerante sem açúcar. Este produto deve ter no rótulo o } \\
\text { termo light ou diet? }\end{array}$ & 100 \\
\hline & $\begin{array}{l}\text { 4- Qual é o perigo em consumir um produto diet sem possuir } \\
\text { restrição alimentar? }\end{array}$ & 100 \\
\hline & 5- Qual é a principal diferença entre o alimento light e odiet? & \\
\hline \multirow{4}{*}{$\begin{array}{l}\text { Quarto encontro: } \\
\text { Transgênico e } \\
\text { Irradiado }\end{array}$} & $\begin{array}{l}\text { 1- Um alimento irradiado é perigoso para o ser humano? Ele } \\
\text { é radioativo? }\end{array}$ & 100 \\
\hline & $\begin{array}{l}\text { 2- Cite pelo menos três exemplos de alimentos que podem } \\
\text { passar pelo processo de irradiação. }\end{array}$ & 100 \\
\hline & $\begin{array}{l}\text { 3- Existe alguma restrição alimentar em relação ao consumo } \\
\text { de alimentos irradiados? }\end{array}$ & 100 \\
\hline & $\begin{array}{l}\text { 4- Um garoto foi ao supermercado e não comprou uma } \\
\text { pipoca com milho transgênico porque acha que é perigoso. } \\
\text { Ele fez certo ou errado? Justifique. }\end{array}$ & 100 \\
\hline
\end{tabular}

Fonte: Dados da pesquisa (2017).

\section{Cartilha}

A cartilha elaborada sobre os termos abordados durante as palestras ficará permanentemente disponível na biblioteca para acesso de todos os alunos da escola. Por 
ser desenvolvida na forma descontraída de quadrinhos (Figura 2), acredita-se que irá despertar interesse por parte daqueles que não absorveram o conteúdo em sua totalidade durante as palestras. Além do alcance aos estudantes que não participaram do projeto, disseminando o conhecimento sobre o assunto para os outros membros da sociedade.

Figura 2: Modelo da história em quadrinhos elaborada na cartilha.
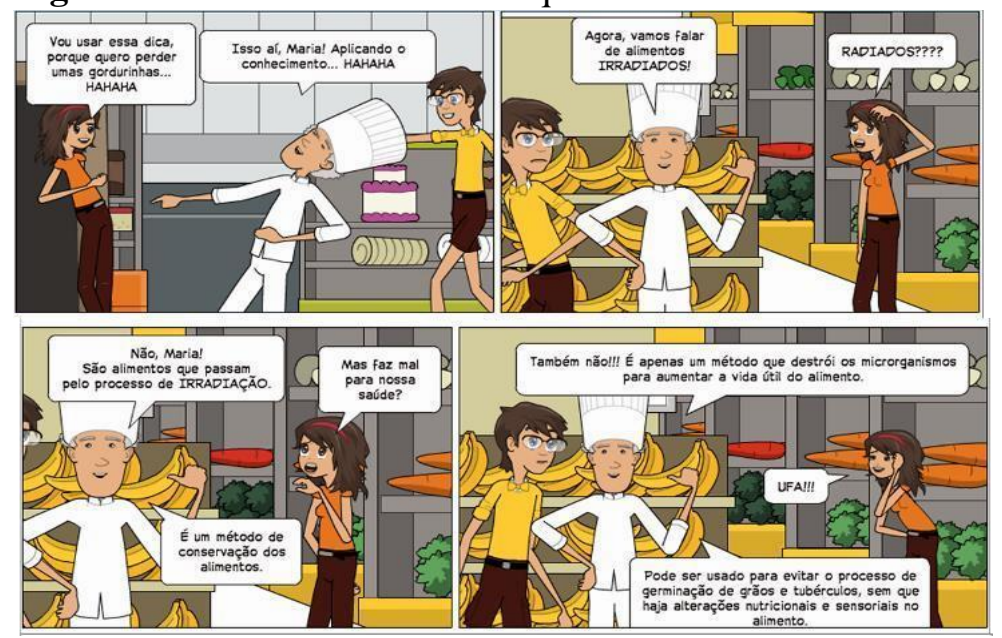

Fonte: Elaborada pelos Autores (2017).

\section{CONCLUSÃO}

O projeto proporciona a conscientização em relação às terminologias e símbolos alimentícios empregados nos rótulos dos produtos e, logo, o estímulo para um consumo mais adequado dos alimentos, hábitos alimentares mais saudáveis e maior autonomia no momento da compra, evitando compras inadequadas e reduzindo o gasto com compras errôneas, principalmente, por aqueles que possuem doenças que exigem alguma restrição alimentar. Por isso, há a necessidade de divulgar informações nas escolas sobre o significado dos termos alimentares. Maior incentivo do governo para isso é necessário para a realização desses projetos para os estudantes aprenderem a interpretar as informações contidas nos rótulos dos alimentos.

Do ponto de vista acadêmico, o projeto proporciona a oportunidade de estudantes disseminarem conhecimentos para a comunidade, bem como obterem novos conhecimentos ao lecionar as aulas. Em relação ao âmbito profissional, é possível aplicar conhecimentos adquiridos ao longo do curso de graduação, além de desenvolver didática e oratória durante as aulas expositivas. Quanto ao campo pessoal, o projeto proporciona o convívio e interação com os alunos para promover o conhecimento, a fim de capacitá-los para repassar o aprendizado para os familiares, amigos e pessoas mais próximas. Ao contribuir para a comunidade, mesmo que em número pequeno, o projeto 
ultrapassa as barreiras da sala de aula, demonstrando o quanto é importante para os alunos serem consumidores conscientes e, a partir disso, ter maior autonomia ao comprar os alimentos de acordo com informações obtidas e suas necessidades. 


\section{REFERENCIAS}

ANTUNES, A. E. C.; MARASCA, E. T. G.; MORENO, I.; DOURADO, F. M.;

RODRIGUES, L. G.; LERAYER, A. L. S. Desenvolvimento de buttermilk probiótico. Ciência e Tecnologia de Alimentos, v. 27, n. 1, p. 83-90, 2007.

BRASIL, 1999. Ministério da Saúde. Política Nacional de Alimentação e Nutrição Portaria no 710, de 10 de junho de 1999. Brasília, 1999.

CAVADA, G. S.; PAIVA, F. F.; HELBIG, E.; BORGES, L. R. Rotulagem nutricional: você sabe o que está comendo? Braz. J. Food. Technol., IV SSA, p. 84-88, 2012.

CURY, A. Transgênicos são $93 \%$ da área plantada com soja, milho e algodão. São Paulo, 2016. Disponível em:

$<$ http://g1.globo.com/economia/agronegocios/noticia/2016/08/transgenicos-sao-93-daarea-plantada-com-soja-milho-e-algodao.html>. Acesso em: 05 jul. 2017.

FORTES, R. C.; MUNIZ, L. B. Efeitos da suplementação dietética com frutooligossacarídeos e inulina no organismo humano: estudo baseado em evidências. Com. Ciências saúde, v. 20, n. 3, p. 241-252, 2009.

JARDIM, F. B. B; FARIA, G. A; DIAS, L. C. F. C.; AFONSO, A. L. T. Rotulagem de alimentos: avaliação e orientação às indústrias e aos consumidores quanto aos aspectos legais e informativos dos rótulos. Boletim Técnico IFTM, v. 1, p. 26-29, 2016.

LOUZADA, M. L. C.; MARTINS, A. P. B.; CANELLA, D. S.; BARALDI, L. G.; LEVY, R. B.; CLARO, R. M.; MOUBARAC, J.; CANNON, G. e MONTEIRO, C. A. Alimentos ultraprocessados e perfil nutricional da dieta no Brasil. Revista Saúde Pública. São Paulo, SP, v. 49, n. 38, jul. 2015.

MATTA, C. M. B. DA; KUNIGK, C. J. Probióticos e prebióticos. Revista Funcionais Nutracêuticos, 2009.

NUNES, M. M. A.; FIGUEIROA, J. N.; ALVEZ, J. G. B. Excesso de peso, atividade física e hábitos alimentares entre adolescentes de diferentes classes econômicas em Campina Gande (PB). Revista Associação de Médica Brasileira, São Paulo, SP, v. 53, n. 2, 2007.

OLIVEIRA, L. L.; BOCCHINI, M. O. Legibilidade visual para informação nutricional em rótulos de alimentos. Blucher Design Proceedings, Brasília, v. 2, n. 2, p. 1-10, ago./set. 2015.

ORNELlAS, C. B. D.; GONÇALVES, M. P. J.; SILVA, P. R.; MARTINS, R. T. Atitude do consumidor frente à irradiação de alimentos. Ciência e Tecnologia de Alimentos, v. 26, n. 1, p. 211-213, 2006.

PONTES, T. E.; COSTA, T. F.; MARUM, A. B. R. F.; BRASIL, A. L.; TADDEI, J. A. A. C. Orientação nutricional de crianças e adolescentes e os novos padrões de consumo: propagandas, embalagens e rótulos. Revista Paulista de Pediatria, v. 27, n. 1, p. 99- 105, 2009. 
SANTOS, C. M. B.; ARAÚJO, C. C.; SOARES, M. B.; JESUINO, R. S. A.; MORAIS, C. C. Experiência de extensão: "Rotulagem nutricional: conheça o que você consome". Revista Ciência em Extensão, v.12, n.4, 2016.

SOUZA, M. F. C.; ARAÚJO, V. F. Adequação do consumo e evolução antropométrica após educação nutricional de pacientes com diabetes mellitus tipo 2. Demetria:

alimentação, nutrição \& saúde, Aracajú, v. 10, n. 1, p. 159-172. 2015.

TEZOTTO-ULIANA, J. V. et al. Radiação gama em produtos de origem vegetal. Revista Virtual de Química, Niterói, v. 7, n. 1, p. 267-277, 2015.

VIEIRA, A. C. P; CORNÉLIO, A. R. ÂMBITO JURÍDICO. Produtos light e diet: o direito de informação ao consumidor. Disponível em:

<http://www.ufrgs.br/agronomia/manualcap1.htm>. Acesso em: 13 de abril. 2018. 